\title{
The Effect of Dietary Structure on the Health Status of Athletes
}

\author{
$\mathrm{Gu}$ Zichao, ${ }^{1, *}$ \\ ${ }^{1}$ Huawei International School, Shaoxing, China, 31200 \\ *Corresponding author. Email: info@ chinadailypack.com

\begin{abstract}
In modern times, more and more people advocate vegetarianism, they believe that a diet without meat will be healthier. The research topic of this paper is whether a vegetarian diet is good for athletes and compared with non-vegetarian diets. This paper makes a systematic literature review by using CNKI, and the research object is athletes. The final result of the study is that vegetarians have a low content of saturated fat, which can reduce blood pressure and cholesterol. The conclusion of this study shows that plant-based diet provides good physical and environmental health benefits, but there is still a lack of experimental research on vegetarianism to further the study.
\end{abstract}

Keywords: vegetarians, health, athletes, blood pressure, diet

\section{INTRODUCTION}

Vegetarian can reduce the risk of chronic diseases, and in performance and recovery benefits. However, the literature on vegetarianism is controversial and confusing.Traditionally, vegetarian or vegetarian diet is considered to be some micronutrients (iron, zinc, calcium, iodine, vitamins $\mathrm{A}, \mathrm{B} 2, \mathrm{~B} 12$ and $\mathrm{D}$ ), and protein, omega-3 fatty acids and total energy requirements. However, vegetarian diet typically contains high levels of composite carbohydrates, dietary fibers, magnesium nitrates, folic acid, vitamins $\mathrm{C}$ and $\mathrm{E}$, carotenoids and other plant chemicals, which may have some benefits to athletes [1]. In view of numerous literature, especially vegetarian, vegetarian, vegetarians, especially in the elite level, further research is to determine the possible differences in the highest training and sports performance. The research topic of this paper is whether a vegetarian diet is good for athletes and compared with non-vegetarian diets. The research method used literature research method and empirical research method. Vegetarian research is beneficial to people's health. With the development of modern society and the rapid growth of population, the demand for animals is also increasing, and vegetarianism is conducive to reducing the demand for animals. More people may become vegetarians in the future, but vegetarians may be short of SHAO protein.

\section{STUDY THE HEALTH STATUS OF MODERN AND PAST VEGETARIANS}

\subsection{The health status of modern vegetarians}

Endurance movement requires healthy and balanced diet. This case report shows the survey results of the ultra-ferrous material (three iron, $11.4 \mathrm{~km}$ swimming, cycling 540 kilometers, $126 \mathrm{~km}$ from the $41 \mathrm{~h}, 18$ minutes by car) Based on the residence and completion of vegetarian food. To this end, the use of echocardiography and spiral studies, 10 Ironman Tri of 10 Ironman Tri, similar ageds, were studied using ultrasound and spiral solutions. In addition, blood samples are removed from the vegan athletes, all in sports season and off-season. Vegetarian athletes have no signs of diet defects or health damage. Compared with the control group, the vegetarian athlete showed a higher oxygen intake at the respiratory compensation point. This situation indicates that even the THE TOTHER IRONMAN's top sports performance is even a vegetarian diet. Whether vegetarian diet is the performance or disadvantage of the performance of the endurance athlete is still an open problem [2]. The purpose of this study is to find anygabach to find a diet in terms of muscle strength and strength in the impact of table tennis players. 183 table tennis players (right handed) were included (70 vegetarian, 113 non-vegetarian) in whom hand grip strength was determined by spring type isometric hand grip 
dynamometer. Although the positive common relationship is obtained when gripping the dominant and non-competitor's strengths in the hands of vegetarians and non-competitors, it has no statistical significance in statistically. In conclusion a well planned vegetarian diet is recommended for an athlete because of its antioxidant properties [3].

Modern Indian female athletes generally existently existently, affecting the nutritional status and exercise performance of the subject. Sixty-four women's national athletes participated in the national camps in the age group of the 16-25 years to participate in this study. Human measuring Viz; uses height, weight, body fat, and slimming weight, and evaluates dietary intake mode through a 24-hour recall method. The performance is evaluated by grading motion until exhausted. Blood samples were collected before exercise to assess hemoglobin. The results indicated that Indian sports women were higher than whey and Ovolacto vegan. They all have no pure vegan. The lactin was significantly higher than non-vegetarians and Ovolacto veggies. There was no striking difference in energy and carbohydrate intake between three groups. Compared with Ovolacto vegetarian and whey protein and wheyine and cream intake, protein intake is significantly higher than vegan, while whey protein vegetarian has a high fat intake $(\mathrm{P}<0.01)$. B-Complex vitamins, iron intake, hemoglobin concentration $(\mathrm{P}<0.05)$ is higher than non-food. The calcium and fiber intake is high, containing propylene-vegetarians $(\mathrm{P}<0.05)$. The endurance and recovery time are better than other groups $(\mathrm{P}<0.05)$. This study indicates that the universality of Non-eating in India's national sports is higher than that of whey wild vegetarians. Some nutrient intake, hemoglobin levels and endurance times are better than vegan, not breast or ovolacto vegan [4].

\subsection{The health status of past vegetarian}

Vegetarianism is a practice that does not eat meat, and the records of records can be traced back to ancient Greece. Until recently, researchers have begun to conduct empirical investigations related to vegetarianism. Power shadow memories Gladiator, difficult training, endurance and efficiency fatal ideas: a flawless war machine. Historically, the hero is a fighter, and a sport Gladiator drugs may be the first form of organized sports medicine. Roman paintings and statues tell us this amazing world warriors. There are traces of a famous Gladiator's known all over the world, in Roman times, similar to our Muhammad Ali or Mike Tyson. Most of them grew up in Italy in the battle school, best known Capua, near Naples: Spartacus, who inflicted a terrible defeat on the Roman military rebel fighter from there.Corner Fighters must endure long-term training courses to play games. Considering the strength of the modern athlete's diet, we should expect to Gladiator has a high protein diet.However, their analysis of these bones has proposed a hypothesis that is a vegan athlete: In the description of the Roman historian Pinsini, he called "hordearii" (barley food) [5]. Plants contain a higher level of ruthenium than animal tissue. The strontium that consumes more plants and less meat will establish a higher level of measurable in the bones. In the horizontal level, the fighter is higher than the previous previous bones [5]. Roman army soldiers, the "Legion", the energy consumption can be estimated about 5,000 kcal per day Veterans executive engineer of work and Legion $6000 \mathrm{kcal}$ of hostilities in. Currently, only workers and athletes reached such a level of energy consumption [5]. The Legion can endure the long-term war election endless "Magnis Itineribus" (forced March), which has incredible fatigue resistance. The daily ration of Legionella $78 \%$ carbohydrate, mainly from wheat or barley. The advantage of this diet provides slow absorption of carbohydrates in order to provide a high energy, easy to digest. It provides a good intestinal ballast and restore energy reserves with organisms [5]. In the ancient world's best fighter is basically strength training or training athletes is higher than the increase in vegetarian. Protein requirements sedentary people. However, the US Institute of Medicine concluded that increased physical activity for the individual needs of the evidence is not compelling, and recommendations for $0.8 \mathrm{~g}$ daily recommended intake of protein per kilogram of body weight (RDA) for use by healthy adults or engage in endurance sports [5]. Whether the impact of vegetarianism is also considered to be related to sports. Observational study vegetarian and non-vegetarian athletes found that the difference in performance or fitness and the amount of animal protein consumption is associated. Short-term intervention studies in which subjects received a vegetarian or vegan diet test period (2-6 weeks) was also detected no difference in performance parameters based on the presence or absence of animal tissue. These survey results and conclusions consistent with previous comments, science and literature is a planned vegan, change to meet the needs of athletes, because it is a Roman corner or veterans [5].

\section{VEGETARIAN DIET PRACTICE AND ENDURANCE PERFORMANCE}

Vegetarian diet is a dietary plan, which has almost no animal protein, so there is no meat in the menu, mainly vegetative food. Vegetarian diet is composed of foods mainly from plants, including: glutinous potato, beans, vegetables, fruits, nuts, etc. Pure veggies are only eaten with botanical foods, no animal proteins or animal by-products, even eggs, milk, honey. Half-eater is mainly edible plant food, but the recipe may include chicken or fish, dairy products and eggs. Vegetarian diet can provide healthy body and produce good benefits for environmental health protection. We think that meat and 
other animal proteins are considered an indispensable part of athletes, resulting in some people questioning some people Can plant diet can sufficient support for motion. Although the protein is still controversial for the protein demand for athletes, the research shows that the strength of the endurance athlete is $1.0-1.5 \mathrm{~g} / \mathrm{kg} / \mathrm{day}$, according to the type and intensity of the training, the intake and carbohydrates in the body calories. For athletes who eat vegetarian, the demand for proteins may be slightly higher. This is mainly due to the low digestibility of food, which is caused by the low energy density and the quality of protein. However, athletes that require high energy intake may find that it is difficult to maintain energy balance only by pure vegetarian food [6].

The balanced intake of macro and micronutrients is essential for conditioning, fatigue recovery after exercise and prevent injury. More and more athletes use vegetarian diet for one or more causes, actually no evidence that the vegetarian diet is beneficial or adversely affecting the performance. Therefore, it is well known that suitable planned vegetarian diet can provide sufficient nutrient energy, with appropriate carbohydrate, fat and protein intake to support performance and health. On the other hand, some investigators have proposed a problem about the unique risk of vegetarian diet, including oligoma and amenorrhea, iron deficiency, lack of vitamin B12, vitamin D lack and mineral disorders [6]. Some rails and live athletes have special diet aimed at improving health and/or performance. It is recommended that the dietary evidence-based method minimizes the risk associated with unnecessary dietary restrictions, which may cause greater damage [7].

The impact of different fat, protein and carbohydrate (CHO) levels, training habits and lifestyle patterns make the dietary interpretation of the specific effects of endurance performance. Typically, the depletion period during prolongation, hard-resistant exercise is stored with low muscle sugar. Urges athletes in training to consume $70 \%$ of Calories as $\mathrm{CHO}$ to maximize the body-cho store. One defensiveness in animal products emphasized in high $\mathrm{CHO}$ plant food will promote athletes in compliance with nutritional recommendations. If you use restriction vegetarian diet, some female athletes may increase their lack of iron deficiency and / or amenorrhate. In general, the highest quality of the vegetarian diet can help endurance athletes maximize body glycogen storage in heavy training, thereby achieving ability. Balanced vegetarian diet provides athletes with a reduction in champion risk factors, while meeting all known nutritional needs [8].

\section{PHYSICAL HEALTH AND VEGETARIANISM: IS THERE A LINK?}

The evidence is not necessary to support the beneficial effects of vegetarian diet on physical properties, especially when controlling carbohydrate intake. Worry has been proposed, emphasizing plant foods to enhance carbohydrate intake and optimizing glycogen reserves may result in increased increase in dietary fibers and cyanic acid to reduce the bioavailability of several nutrients, including zinc, iron and some other trace Mineral. However, there is no convincing evidence that vegetarian athletes suffer damaged nutritional status in its interaction of their heavy fatigue and plant-food diet practice, which is affected by performance, health or damage. Although there are some concerns about the protein intake of vegetarians, the data shows that all the necessary and unnecessary amino acids can be provided alone using a variety of foods and energy intake. Some people are concerned that vegetarian women are increasing the risk of oligomeric proteins, but evidence shows that low energy intake, not the quality of diet is the main reason. In short, the vegetarian diet itself is independent of the improved aerobic endurance performance. Although the nutritional status of vegetarian athletes has been proposed, the nutritional conditions of a variety of vegetarian diet are especially compatible with successful sports. [9]

So far, the advantages and disadvantages of venue food diet have not been fully investigated. If there is exercise performance, it is necessary to study the potential benefits of long-term vegetarian diet. Plan a good breast ovary or vegetarian diet will meet the nutritional needs of most athletes. However, vegetarian or vegetarian diet may provide insufficient energy and fail to meet daily nutritional needs. It should include daily, nutrient, energy-dense vegetarian replacement to ensure sufficient energy and nutrient intake. Most vegetnces of high carbohydrate content is conducive to recovery and maintain a sufficient glycogen in athletes stored in high daily carbohydrates. Although animal food is a good source of protein, iron, zinc, and vitamin B12, dairy products have rich source of calcium, and vegetarians can access alternative sources. The source of food with vitamin B12 should be included in vegetarian. Further research is needed to better understand the influence of long-term vegetarian diet on menstruation in female athletes [10].

\section{CONCLUSION}

In conclusion, the chemicals contained in a vegetarian diet have some benefits for athletes, and given the paucity of literature comparing omnivorous, vegetarian and vegan athletes (especially at the elite level), further research is necessary to determine the 
differences that may occur in training and athletic performance at the highest levels. But athletes are advised to plan a well-planned vegetarian diet because of its antioxidant properties. The conclusion that the previous review of scientific literature is, carefully planned, diverse vegetarian diet to meet the needs of athletes. Although some people have some concerns about the nutritional status of the puree, diversified and careful planned vegetarian diet is matched to successful exercise efforts. So far, the advantages and consistency of athletes have not yet been fully investigated. so in the future, scientists can make some explorations in diet to make people athletes healthier and push the limits. People can also avoid some diseases, such as cancer, by eating a proper diet.

\section{AUTHORS’ CONTRIBUTIONS} $\mathrm{Gu}$

This paper is independently completed by Zichao

\section{ACKNOWLEDGMENTS}

In the process of writing this paper, I benefit from the existence of the students. They generously helped me collect the information I need and put forward a lot of valuable suggestions. Thanks to their help, there is no help, this paper will not have today's achievements.

I were particularly grateful to my teacher, and he guided me to complete this paper. He carefully read the whole draft and put forward hard and valuable criticism. His academic excellent standards make my revision and exciting and gratifying experience. I would also like to thank my classmates and friends Xie Tianhe Zhang Yi, and their smart ideas and keen observations are very constructive.

When I wrote this papers, my parents took care of my life and gave me a lot of encouragement. They are always working with me.I appreciated it very much.

\section{REFERENCES}

[1] Kenneth Vitale; Shawn Hueglin, Update on vegetarian and vegan athletes: a review: Review Article [J] The Journal of Physical Fitness and Sports MedicineVolume 10, Issue 1, 2021, PP $1-11$.

[2] Roman Leischik; Norman Spelsberg; Birgit Dworrak; Melchior Seyfarth, Tripple-Ironman Based on Vegan Raw Vegetable/Fruits Diet: Physiological Echocardiographic and Blood Analysis Findings [J] American Journal of Sports ScienceVolume 1, Issue 4, 2014.

[3] K. V. Vishrutha; Arati P Rao; K.G. Prarthana; U Chaitra, Evaluation of Hand Grip Strength in Vegetarian and Non Vegetarian Table Tennis
Players [J] Indian Journal of Clinical Anatomy and PhysiologyVolume 3, Issue 1. 2016, PP 83-85.

[4] Khanna G L; Lal P R; Kommi K; Chakraborty T, A Comparison of a Vegetarian and Non-Vegetarian Diet in Indian Female Athletes in Relation to Exercise Performance [J] Journal of Exercise Science and Physiotherapy, Volume 2, Issue $1 \& 2$. 2006. PP 27-34.

[5] Borrione, Paolo; Grasso, Loredana; Quaranta, Federico; Parisi, Attilio,Vegetarian diet and athletes: FIMS Position Statement 2009 [J] International SportMed JournalVolume 10, Issue 1, 2009, PP 53-60.

[6] Umile Giuseppe Longo; Filippo Spiezia; Nicola Maffulli; Vincenzo Denaro, THE BEST ATHLETES IN ANCIENT ROME WERE VEGETARIAN! [J] Journal of Sports Science and MedicineVolume 7, Issue 4, 2008.

[7] Larson-Meyer, Dietary Practices Adopted by Track-and-Field Athletes: Gluten-Free, Low FODMAP, Vegetarian, and Fasting [J] International Journal of Sport Nutrition and Exercise Metabolism Volume 29, Issue 2. 2019, PP 236-245.

[8] Nieman D C, Vegetarian dietary practices and endurance performance.Journal [J] The American Journal of Clinical Nutrition Volume 48, Issue 3 Suppl. 1988. PP 754-61.

[9] Nieman D C, Physical fitness and vegetarian diets: is there a relation? [J] The American Journal of Clinical NutritionVolume 70, Issue 3 Suppl. 1999. PP 570S.

[10] Cox, Gregory R. The vegetarian athlete [J] International SportMed Journal, Volume 2, Issue 2. 2001. PP1-8. 